\title{
A GENERALIZATION OF THE FLAT CONE CONDITION FOR REGULARITY OF SOLUTIONS OF ELLIPTIC EQUATIONS
}

\author{
GARY M. LIEBERMAN
}

\begin{abstract}
Barrier arguments are used to prove regularity of boundary points for a large class of uniformly elliptic operators when the domain satisfies a geometric condition. The condition is that the exterior of the domain contains a suitable lower dimensional set.
\end{abstract}

Although necessary and sufficient conditions are known for the regularity of boundary points for the Dirichlet problem for a uniformly elliptic equation when the coefficients have appropriate global continuity properties (see, e.g., [1]) or when the equation is in divergence form [9], the conditions for general coefficients are not yet fully understood. Counterexamples show that points regular for the Laplacian need not be regular for other uniformly elliptic operators, and vice versa (see [11]), while the recent work of Bauman [3] characterizing regular points for such equations does not seem to present an easily verified condition. However, various simple sufficient conditions for a boundary point to be regular for the class of uniformly elliptic equations are known. We present here a simple sufficient geometric condition (the exterior generalized flat cone condition) for a boundary point to be regular for any uniformly elliptic equation; this condition is satisfied in many cases when the exterior of the domain is only $(n-1)$-dimensional. Our results include most of the well-known geometric conditions such as the cone condition [10] and the flat cone condition [4, p. 165] (only discussed for the Laplace operator in that work). On the other hand, the well-known condition A of Ladyzhenskaya and Ural'tseva $[6$, p. 6] is neither contained in nor contains our condition as we show in the third section of this paper. Another sufficient condition for regularity of boundary points is the Wiener criterion of Landis [7]; however, the relation between that criterion and ours is currently unclear.

Also to be mentioned here is the different approach to constructing barrier functions, in some ways perhaps more significant than the result itself. One such construction creates higher dimensional barriers from lower dimensional ones (see the proof of Lemma 2 below). We wish to thank Professor David Gilbarg for pointing out this idea, which, although implicit in most studies of the mixed boundary value problem (see [2, 8], etc.), seems not to have been explicitly used before. Another means of obtaining barriers is by using a simple geometric observation (see the proof of Theorem 1).

The plan of this work is as follows. In $\S 1$ we define the generalized flat cone condition and provide some preliminary discussion. The sufficiency of this condition is demonstrated in $\S 2$. As boundary regularity follows in a standard way from the

Received by the editors April 5, 1986.

1980 Mathematics Subject Classification (1985 Revision). Primary 31B25, 35B45, 35J67; Secondary 35B65, 35J25. 
existence of an appropriate barrier function, we shall be concerned primarily with the barrier construction. Examples to illustrate our condition and contrast it with other conditions appear in $\S 3$. Some generalizations of our condition and some applications of our barrier extension technique to other conditions are given in $\S 4$.

1. Statement of the condition. For $n \geq 3, x=\left(x^{\prime}, x^{n}\right)=\left(x^{\prime \prime}, x^{n-1}, x^{n}\right)=$ $\left(x^{1}, \ldots, x^{n}\right)$ denotes a point in $\mathbf{R}^{n}$ and $\Omega$ denotes a connected open subset of $\mathbf{R}^{n}$ with nonempty exterior. Since all our hypotheses are invariant under orthogonal changes of coordinates, we assume that the point 0 lies in the boundary of $\Omega$ and we restrict our attention to a neighborhood of 0 ; in addition we assume that $\Omega$ lies in a standard position which we now explain. We call a subset of the halfspace $\mathbf{R}_{+}^{n}=$ $\left\{x^{n}>0\right\}$ a crinkly halfplane if a continuous branch of the function $\arctan \left(x^{n} / x^{n-1}\right)$ can be defined in the exterior of this set. Note that according to this definition, the halfspace $\mathbf{R}_{+}^{n}$ is a crinkly halfplane.

We say that $\Omega$ satisfies an exterior crinkly halfplane condition at 0 if the intersection of some ball centered at 0 with some crinkly halfplane is exterior to $\Omega$. Before giving the generalized cone condition, we define, for $0<\theta<1$, the map $P_{\theta}$ by

$$
P_{\theta}\left(x^{\prime}, x^{n}\right)=\left(x^{\prime}, x^{n}-\theta|x|\right) \text {. }
$$

A generalized flat cone is the preimage under some $P_{\theta}$ of a crinkly halfplane. The exterior generalized flat cone condition is then defined analogously to the exterior crinkly halfplane condition.

Our differential operators will all have the form

$$
L=a^{i j} D_{i j}+b^{i} D_{i}
$$

where $a^{i j}$ and $b^{i}$ are locally bounded functions defined in $\Omega$ with $a^{i j}=a^{j i}$, and the convention that repeated indices are summed from 1 to $n$ is observed. We also assume that the matrix $A=\left(a^{i j}\right)$ satisfies the inequality

$$
|\xi|^{2} \leq a^{i j} \xi_{i} \xi_{j} \leq \Lambda|\xi|^{2}
$$

for some (positive) constant $\Lambda$ and all vectors $\xi$ in $\mathbf{R}^{n}$. (The first inequality in (1.2) is a normalization made for notational simplicity only.) Assumptions on the coefficients $b^{i}$ will be made below. For suitable functions $c$, operators of the form $L+c$ also fall within our framework, but for technical reasons we defer the discussion of such operators to $\S 4$.

The key step here is to combine an idea used in [8] with a barrier construction from [10]. The results of this construction are summarized in the following lemma.

LEMMA 1. Let $K$ be a subset of $\mathbf{R}^{2}$ on which polar coordinates $r$ and $\theta$ can be introduced with $0<\theta<\theta_{0}$ and $r \leq h$ in $K$ for positive constants $h$ and $\theta_{0}$. Let $L$ have the form (1.1) where the functions $a^{i j}$ satisfy (1.2) in $K$, and suppose that there is a nonnegative constant $\Lambda_{0}$ such that

$$
|b| \leq \Lambda_{0} / r \text { in } K .
$$

Then there is a positive constant $\tau_{0}$ determined only by the numbers $\Lambda, \theta_{0}$, and $\Lambda_{0}$ such that for each $\tau$ in the interval $\left(0, \tau_{0}\right)$, there is a positive $C^{2}\left[0, \theta_{0}\right]$ function $f$ (determined only by $\tau, \Lambda, \theta_{0}$, and $\Lambda_{0}$ ) such that $w_{0}\left(x^{1}, x^{2}\right)=r^{\tau} f(\theta)$ obeys the estimate

$$
L w_{0} \leq-r^{\tau-2} \text { in } K
$$


It is important to observe that only the sizes of the coefficients of $L$ are involved in this lemma.

2. The main result. In this section we demonstrate the continuity of the Wiener generalized solution to the Dirichlet problem for an elliptic equation wherever an exterior generalized flat cone condition is satisfied. As a first step we construct a barrier for domains satisfying a crinkly halfplane condition.

LEMMA 2. Suppose that $\Omega$ satisfies an exterior crinkly halfplane condition at 0 and let $L$ have the form (1.1) with the coefficients $a^{i j}$ satisfying (1.2) for some positive constant $\Lambda$. Suppose also that there is a nonnegative constant $\Lambda_{0}$ such that

$$
|b(x)| \leq \Lambda_{0} /|x|
$$

for any $x$ in $\Omega$ with $|x| \leq 1$. Then there are a positive constant $K$ and a function $w$ such that

$$
\begin{gathered}
L w(x)<0 \quad \text { if } x \text { is in } \Omega \text { and }|x|<K, \\
w(x)>0 \quad \text { if } x \text { is in } \bar{\Omega} \text { and } 0<|x|<K, \\
w(0)=0 .
\end{gathered}
$$

PROOF. The desired function is given by

$$
w(x)=w_{0}\left(x^{n-1}, x^{n}\right)+\mu\left|x^{\prime \prime}\right|^{2}
$$

for a sufficiently small constant $\mu$. A straightforward calculation shows that (2.2a) is valid while $(2.2 \mathrm{~b})$ and $(2.2 \mathrm{c})$ are clear.

The usual maximum principle argument (see, e.g., [10, p. 98]) implies the regularity of 0 if $\Omega$ satisfies an exterior crinkly halfplane condition there. Rather than state the regularity result precisely for the crinkly halfplane condition, we proceed directly to the generalized flat cone condition.

THEOREM 1. Suppose that $\Omega$ satisfies an exterior generalized flat cone condition at 0 , let $L$ have the form (1.1) with the coefficients $a^{i j}$ satisfying (1.2) for some positive constant $\Lambda$, and let $\tau$ be a positive constant. Suppose also that there is a nonnegative constant $\Lambda_{0}$ such that (2.1) holds for any $x$ in $\Omega$ with $|x| \leq 1$. If $\Phi$ is continuous at 0 and if $f$ is a locally bounded function such that $f(x)=\bar{O}\left(|x|^{\tau-2}\right)$ near 0 , then the Wiener generalized solution of the Dirichlet problem

$$
L u=f \quad \text { in } \Omega, \quad u=\Phi \quad \text { on } \partial \Omega
$$

(if it exists) is continuous at 0.

ProOF. First, without loss of generality, we may assume that $0<\tau<\tau_{0}$. We then observe that the change of variables $y^{\prime}=x^{\prime}, y^{n}=x^{n}-\theta|x|$ does not affect the form of the operator $L$. Specifically if $w_{1}(x)=w_{0}\left(y^{n-1}, y^{n}\right)$, we have $L w_{1}=\alpha^{i j} D_{i j} w_{0}+\beta^{i} D_{i} w_{0}$, where

$$
\begin{gathered}
\alpha^{n-1, n-1}=a^{n-1, n-1} /(1-\theta), \\
\alpha^{n-1, n}=\alpha^{n, n-1}=\left[a^{n, n-1}-\theta a^{i, n-1} x^{i} /|x|\right] /(1-\theta), \\
\alpha^{n n}=\left[a^{n n}-\theta a^{n n} x^{n} /|x|+\theta^{2} a^{i j} x^{i} x^{j} /|x|^{2}\right] /(1-\theta), \\
\beta^{n-1}=b^{n-1} /(1-\theta), \\
\beta^{n}=\left[b^{n}-\theta b^{i} x^{i} /|x|-\theta a^{i i} /|x|+\theta a^{i j} x^{i} x^{j} /|x|^{3}\right] /(1-\theta) .
\end{gathered}
$$


The only condition not immediately obvious is that

$$
\alpha^{i j} \xi_{i} \xi_{j} \geq|\xi|^{2}
$$

for $\xi=\left(\xi_{n-1}, \xi_{n}\right)$. Setting $\xi^{\prime \prime}=0$ and $\zeta=\xi-\theta \xi_{n} x /|x|$, we see that $\alpha^{i j} \xi_{i} \xi_{j}=$ $a^{i j} \zeta_{i} \zeta_{j} /(1-\theta)$ and that $|\zeta| \geq(1-\theta)|\xi|$. Therefore (1.2) implies (2.4) and hence the function $w$ given by $w(x)=w_{0}\left(y^{n-1}, y^{n}\right)+\mu\left|x^{\prime \prime}\right|^{2}$ is a barrier for $L$ and $f$ as defined in [10], and the theorem is proved.

Note that the Wiener generalized solution exists if, e.g., the functions $a^{i j}, b^{i}$, and $f$ are locally Hölder continuous. These smoothness hypotheses can be relaxed somewhat; see $\S 4$ for details.

3. Examples. We now examine some specific examples of the generalized flat cone and crinkly halfplane conditions.

The simplest example for these conditions is a convex domain, for which our crinkly hyperplane is the halfspace on the opposite side of a supporting hyperplane to the domain. More generally, if a domain satisfies an exterior cone condition at a boundary point, it satisfies an exterior generalized flat cone condition there. First observe that the cone can be rotated and translated so that the positive $x^{n}$-axis coincides with the axis of the cone and the vertex of the cone is the origin. Without loss of generality, the exterior cone may be assumed convex, and thus the equation of the surface of the cone will be $x^{n}-\theta|x|$ for some constant $\theta$ with $0<\theta<1$. Using the map $P_{\theta}$ for this constant $\theta$ shows that the domain satisfies an exterior generalized flat cone condition (with the corresponding crinkly halfplane being the halfspace $\mathbf{R}_{+}^{n}$ ).

As defined in [4], a domain satisfies an exterior flat cone condition if there is a cone with vertex at a given point on the boundary and a hyperplane containing the axis of the cone such that the exterior of the domain includes the intersection of the hyperplane with the interior of the cone. If the equation of this cone is as before, then we proceed as in the case of an exterior cone except that now the corresponding crinkly halfplane is the intersection of the given hyperplane with the halfspace $\mathbf{R}_{+}^{n}$.

The crinkly halfplane in the crinkly halfplane condition can be quite bizarre: any surface which can be represented as the graph of a continuous function $x^{n-1}=$ $\phi\left(x^{\prime \prime}, x^{n}\right)$ with $\phi\left(x^{\prime \prime}, 0\right)=0$ is a crinkly halfplane.

Next we observe that the set of points in $\mathbf{R}^{n}$ for which $x^{n-1} \neq 0$ or $x^{n}>0$ satisfies an exterior generalized flat cone condition at each point of its boundary but not condition A of Ladyzhenskaya and Ural'tseva because the complement of this set has Lebesgue measure zero.

Finally we give an example of a domain which does not satisfy an exterior generalized flat cone condition at the origin but for which condition $\mathrm{A}$ is satisfied. For each positive integer $k$ let $\Omega_{k}$ be the closed ball of radius $1 / 4 k$ centered at the point $(0, \ldots, 0,1 / k)$ and let $\Omega$ be the complement in $\mathbf{R}^{n}$ of the union of the $\Omega_{k}$ 's. It is easy to check that the intersection of a ball of radius $r$ centered at the origin with the complement of $\Omega$ has volume greater than some fixed multiple of $r^{n}$, so that condition A holds. On the other hand, the generalized flat cone condition is not satisfied because the complement of $\Omega$ is disconnected in any neighborhood of the origin. 
Condition $\mathrm{A}$ is a sufficient condition for regularity of boundary points by virtue of [5, Theorem 9.30]. Thus the last two examples show that neither our flat cone condition nor condition A can be a necessary condition for the regularity of a boundary point for any uniformly elliptic operator.

4. Extensions of the results. So far, we have attempted to follow a middle road between weak hypotheses and simple exposition, with our basic goal being to include the flat cone condition. In this section we look at more complicated situations.

Clearly our hypotheses are invariant under any Lipschitz coordinate change which is $C^{2}$ in a deleted neighborhood of the origin and which has second derivatives degenerating near the origin no faster than $1 /|x|$. In particular our "flat" cone may lie on the surface of a sphere.

Next we observe that the full force of the uniform ellipticity hypothesis (1.2) has not been used. For the crinkly halfplane condition, the precise ellipticity hypothesis can be stated very simply: We need only assume the full matrix $A$ to be positive semidefinite with bounded maximum eigenvalue and the 2-by-2 submatrix $A^{\prime}=\left(a^{i j}\right)_{i, j \geq n-1}$ to have its minimum eigenvalue bounded away from zero. These hypotheses are not affected by an application of the map $P_{\theta}$. Thus when an exterior cone condition is satisfied, it is enough for $A$ to be positive semidefinite with a uniformly bounded maximum eigenvalue provided that, after a suitable translation and rotation taking the axis of the cone to the $x^{n}$-axis, the minimum eigenvalue of $A^{\prime}$ is bounded away from zero.

In a different direction, we observe that the coefficients $b^{i}$ can become infinite as we approach all of $\partial \Omega$; the proof uses a patching argument of Pucci $[13, \S 4]$.

For operators of the form $L+c$, we know from the calculations in [10] that Lemma 1 remains valid provided $c \leq \Lambda_{1} / r^{2}$ for a sufficiently small nonnegative constant $\Lambda_{1}$. Since $w_{0}$ is a positive subsolution for the operator $L+c$, we can use the generalized maximum principle [12, Theorem 10 of Chapter 2] to prove Theorem 1 if we assume that $|c| \leq \Lambda_{1}|x|^{\tau-2}$ for $|x| \leq 1$ and a positive constant $\tau$; in this case, $\Lambda_{1}$ can be arbitrary. Of course, then we have no guarantee that a Wiener generalized solution exists even if the coefficients of $L+c$ are smooth.

We conclude by noting that the Wiener generalized solution exists if $A$ is locally continuous and $b$ is locally bounded and measurable. This solution will lie in the Sobolev space $W_{\text {loc }}^{2, n}$. Because the Bakelman-Aleksandrov-Pucci maximum principle $[5$, Theorem 9.1] applies to functions in this space, our barrier argument is still valid in this case.

\section{REFERENCES}

1. Yu. A. Alkhutov, Regularity of boundary points relative to the Dirichlet problem for second-onder elliptic equations, Math. Notes 30 (1981), 655-660.

2. A. Azzam, Smoothness properties of solutions of mixed boundary value problems for elliptic equations in sectionally smooth n-dimensional domains, Ann. Polon. Math. 40 (1981), 81-93.

3. P. A. Bauman, A Wiener test for nondivergence structure, second-order elliptic equations, Indiana Univ Math. J. 34 (1985), 825-844.

4. K. L. Chung, Lectures from Markov processes to Brownian motion, Springer-Verlag, New York, 1982.

5. D. Gilbarg and N. S. Trudinger, Elliptic partial differential equations of second order (2nd ed.), Springer-Verlag, New York, 1983. 
6. O. A. Ladyzhenskaya and N. N. Ural'tseva, Linear and quasilinear elliptic equations, Academic Press, New York, 1968.

7. E. M. Landis, s-capacity and its application to the study of solutions of a second onder elliptic equation with discontinuous coefficients, Math. USSR-Sb. 5 (1968), 177-204.

8. G. M. Lieberman, Mixed boundary value problems for elliptic and parabolic differential equations of second onder, J. Math. Anal. Appl. 113 (1986), 422-440.

9. W. Littman, G. Stampacchia, and H. F. Weinberger, Regular points for elliptic equations with discontinuous coefficients, Ann. Scuola Norm. Sup. Pisa (3) 17 (1963), 45-79.

10. K. Miller, Barriers on cones for uniformly elliptic operators, Ann. Mat. Pura Appl. 76 (1967), 93-105.

11. , Exceptional points for the nondivergence equation which are regular for the Laplace equation-and vice versa, Ann. Scuola Norm. Sup. Pisa (3) 22 (1968), 315-330.

12. M. H. Protter and H. F. Weinberger, Maximum principles in differential equations (reprinted ed.), Springer-Verlag, Berlin and New York, 1984.

13. C. Pucci, Regolarità alla frontiena di soluzioni di equazioni ellitiche, Ann. Mat. Pura Appl. 65 (1964), 311-328.

Department of Mathematics, IOWA State University, Ames, Iowa 50011 